\title{
Intracellular functions of RNA-binding protein, Musashi1, in stem and cancer cells
}

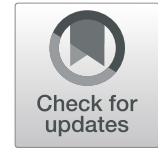

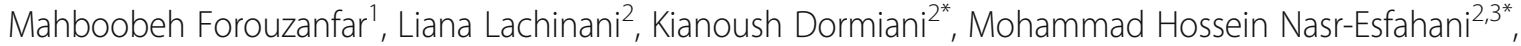 \\ Ali Osmay Gure ${ }^{4}$ and Kamran Ghaedi ${ }^{1,3^{*}}$ (i)
}

\begin{abstract}
RNA-binding protein, musashi1 (MSI1), is a main protein in asymmetric cell division of the sensory organ precursor cells, whereas its expression is reported to be upregulated in cancers. This protein is a critical element in proliferation of stem and cancer stem cells, which acts through Wht and Notch signaling pathways. Moreover, MSI1 modulates malignancy and chemoresistance of lung cancer cells via activating the Akt signaling. Due to the main role of MSI1 in metastasis and cancer development, MSI1 would be an appropriate candidate for cancer therapy. Downregulation of MSI1 inhibits proliferation of cancer stem cells and reduces the growth of solid tumors in several cancers. On the other hand, MSI1 expression is regulated by microRNAs in such a way that several different tumor suppressor miRNAs negatively regulate oncogenic MSI1 and inhibit migration and tumor metastasis. The aim of this review is summarizing the role of MSI1 in stem cell proliferation and cancer promotion.
\end{abstract}

Keywords: Cancer stem cells, Cancer progression, Musashi, RNA-binding protein

\section{Introduction}

Musashi, a neural RNA-binding protein, was identified in 1994 with an important role in neural development. This protein is also essential for asymmetrical division and development of sensory organ in Drosophila melanogaster. Asymmetric cell division of the sensory organ precursor (SOP) cell in wild type animal generates non-neuronal precursor cell and neuronal precursor cell whereas in msi1 mutated animal, it generates two nonneuronal precursor cells. As a result of this symmetrical division in Drosophila, double hair-shape phonotypes emerge. This gene was termed "Musashi" after

\footnotetext{
*Correspondence: k_dormiani@royaninstitute.org; mh.nasresfahani@royaninstitute.org; kamranghaedi@sci.ui.ac.ir

2Department of Molecular Biotechnology, Cell Science Research Center, Royan Institute for Biotechnology, ACECR, Isfahan P.O. Code 816513-1378, Iran

'Department of Cell and Molecular Biology and Microbiology, Faculty of Biological Science and Technology, University of Isfahan, Hezar Jerib Ave., Azadi Square, Isfahan P.O. Code 81746, Iran

Full list of author information is available at the end of the article
}

Miyamoto Musashi, a famous Japanese samurai who was fighting with two swords $[1,2]$. In vertebrates, musashi family have two extremely conserved homolog proteins, MSI1 (Musashi1) and Musashi 2 (MSI2), which were discovered in mice $[3,4]$. MSI2 plays important roles in hematopoiesis, and its dysregulated expression is associated with several hematopoietic malignancies [5]. Further studies revealed that Msi1 is expressed in CNS stem cells and neural stem cells of vertebrates $[6,7]$. Human MSI gene is located on chromosome 12, which encodes a protein containing two tandems with highly conserved RNA-recognition motifs. Each RNA-binding domain (RBD) is composed of antiparallel $\beta$-sheets packed against two $\alpha$-helices. In vitro selection method, SELEX, demonstrated that MSI1 blocks translation of its target genes by binding to (G/A) Un (AGU) sequence motifs $(n=1-3)$ at 3 'UTR of target mRNAs. These motifs are repeated two or three times in 3'UTR regions [8]. The mechanism of MSI1 function is prevention of the initiation of translation of Numb mRNA by competing with eIF4G factor for binding to poly(A) binding 
protein (PABP) and hindering the construction of $80 \mathrm{~s}$ ribosome complex [9]. Therefore, MSI1 protein has an important role in activating Notch signaling by targeting Numb mRNA expression as an inhibitor of Notch pathway [10].

MSI1 targets several genes, which are involved in the proliferation of stem cells and cell cycle regulation. Cancer stem cells undergo symmetric and asymmetric cell divisions. It is demonstrated that MSI1 expression increases proliferation of cancer cells in different type of cancers $[11,12]$. In the normal state, MSI1 expression in mammary epithelial cells drives proliferation of mammary stem/progenitor cells by activation of Notch and Wnt pathways. Downregulation of the cyclin-dependent kinase inhibitor p21 ${ }^{\text {Cip } 1}$, Dickkopf-3 (DKK3), and Numb mRNA followed by expression of MSI1 is responsible for cell proliferation [13]. In this review, we discuss the functional aspects of MSI1 in stem cell biology and cancer development.

\section{The role of $M S I 1$ expression in stem cells}

Early studies have shown that mouse Msi1 is highly expressed in CNS progenitor cells and has an important role in brain development. Expression of Msi1 is also reported in astroglial progenitor cells and mature astrocyte cells $[3,6,7]$. Msi1 is a vital factor for self-renewal maintenance of stem cells. The expression of Msi1 is required for oligodendrocyte progenitor lineage cell survival and preventing differentiation of oligodendrocyte progenitor cells into mature oligodendrocytes [14]. Indeed, regulation of Msi1 function is necessary for transition cell fate in rat neural stem/progenitor cells (NSPCs). Phosphorylation of regulatory conserved site at serine 337 in MSI1 protein causes differentiation of neural stem/progenitor cells and SH-SY5Y cells by accumulation of p21 WAF1/${ }^{C I P 1}$ protein as target mRNA for MSI1. In fact, inhibition of MSI1 protein phosphorylation acts like overexpression of this protein and stop differentiation through regulation of cell cycle inhibitory protein [15].

MSI1 could be used as a stem cell marker to isolate adult stem cells in intestinal epithelium. Plateroti and colleagues developed transgenic mouse model for targeted expression of Msi1 in the intestinal epithelium to study the role of Msi1 in cell cycle and stem cell activity. Expression of stem cell markers Ccnd1, Cdk6, and Sox4 were enhanced as a result of targeted Msi1 overexpression and cell proliferation rate in the intestinal epithelium $[16,17]$. A population of active stem cells which called reserve intestinal stem cells (rISCs) are resistant to $\gamma$ radiation treatment of malignancy. During the regenerative phase after injury induction by $\gamma$ radiation, the expression level of Msi1 increases as an inhibitor of $\mathrm{p} 21^{\text {Waf1/Cip1 }}$ which promotes proliferation of intestinal stem cells and plays a critical role during regenerative responses [18]. As regards MSI1 function in maintenance of stem cell properties and regenerative phase after damage which mentioned above, the role of this gene in regeneration of lost neural cells in neurodegenerative disease could be interesting for investigation in future.

Furthermore, Msi1 is highly expressed in spermatogonia and plays a critical role during germ cell development in mouse. Recently, it has been shown that Msi2 and enhancer of rudimentary homolog (Drosophila) (Erh) are potential targets of MSI1 through three canonical MSI1 binding sites in their 3'UTR. Subcellular relocation of MSI1 during spermatocyte differentiation prevents activation of Msi2 and Erh. Moreover, MSI1 stabilizes Msi2 RNA within the cytoplasm of spermatogonia and represses the translation of Msi2. Nuclear translocation of MSI1 occurs as a result of interaction between MSI1 and importin- $\beta$ nuclear import protein, IPO5, which acts as a unique molecular switch for MSI2 release. Therefore, MSI1 is a major regulator of posttranscriptional control during murine germ cell development $[19,20]$. Consistent with Xenopus findings, MSI1 associates with embryonic poly (A) binding protein family (ePABP) or the canonical somatic cell poly(A) binding protein (PABPC1) and activates translation of target mRNAs in oocyte maturation [21]. Although these studies confirmed that MSI1 is a key component of stem cell development and oocyte maturation, understanding the similar function of MSI1 and its role in human fertility and infertility remains to be obscured. Schematic representation of MSI1 function in stem and cancer stem cells is shown in Fig. 1. In conclusion, a variety of these functions are mentioned in Table 1.

MSI2, another member of MSI RNA-binding protein family, has a critical role in maintenance of hematopoietic stem cells. Rentas and colleagues revealed that MSI2 expression attenuates aryl hydrocarbon receptor (AHR) signaling in hematopoietic stem and progenitor cell (HSPC) [22]. On the one hand, the role of MSI2 has been addressed in chemoresistance ability of liver cancer stem cells. Recent study has shown that there is a positive correlation between MSI2 and LIN28 expressions in maintenance of stemness properties and chemoresistance of hepatocellular carcinoma (HCC) cancer stem cells [23].

\section{Association of MSI1 expression and cancers}

RNA sequencing data have shown that the expression level of MSI1 increased in several types of solid tumors like breast, prostate, lung, and brain tumors while its expression in normal tissues is limited to stem cells [24, 25]. Therefore, it could be concluded that the overexpression of MSI1 enhances cancer promotion. In cervical cancer, MSI1 expression increases cancer cell proliferation through regulation of the cell cycle. MSI1 as an 


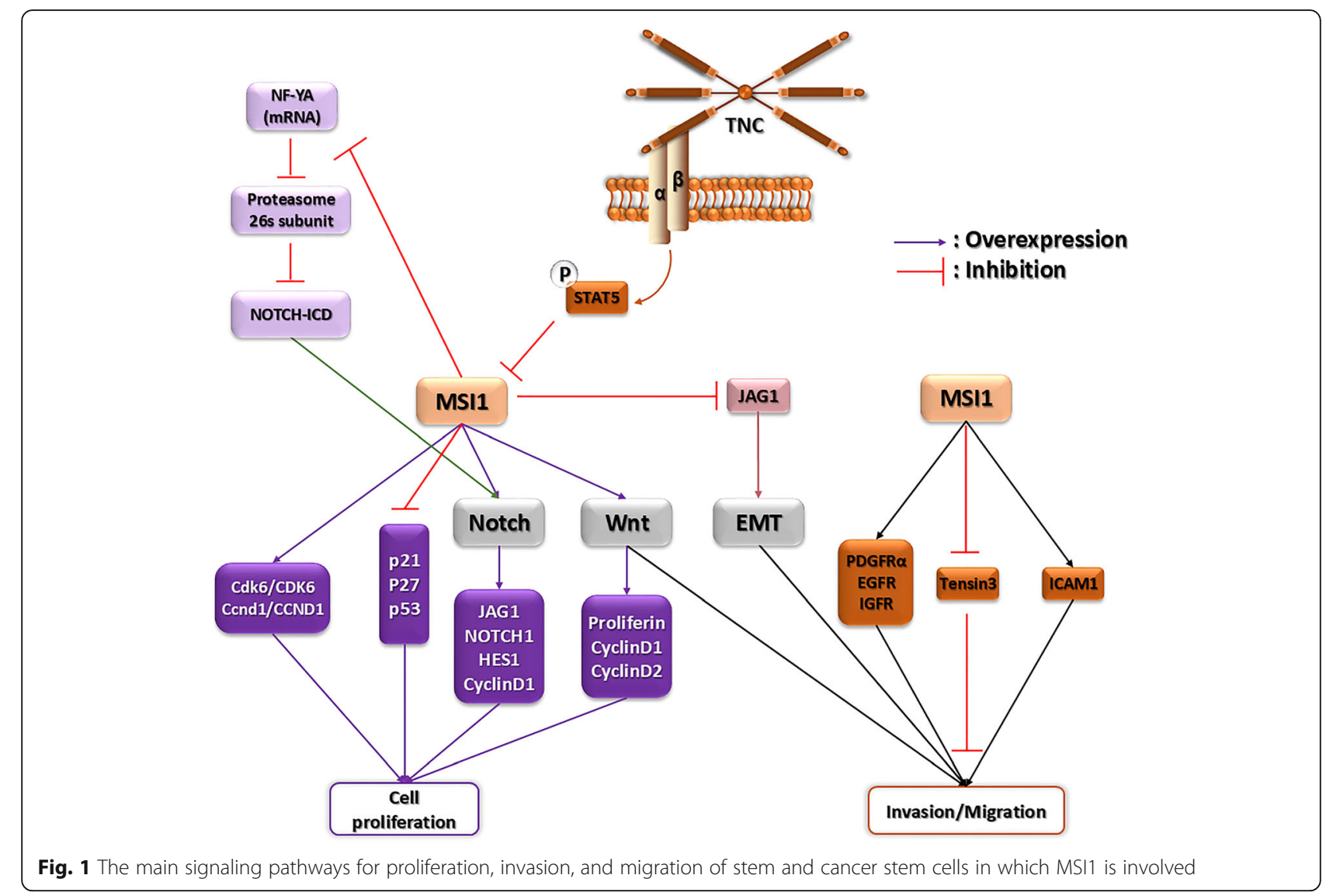

RNA-binding protein directly binds to p21, p27, and p53 mRNAs and prohibits translation of checkpoint regulators. As a result, MSI1 promotes G0/G1 to S phase transition [12]. MSI1 controls the proliferation of cancer stem cells by modulating the Notch and Wnt pathways, as well as Akt signaling pathway. It is reported that in spheroid breast cancer cell culture, expression of MSI1 elevates in $\mathrm{CD}_{133^{+}}$cancer stem cells. By reduction of MSI1 expression in spheroid culture, proliferation of cancer stem cells decreases through reduction of Notch1 and cancer stem cell markers such as Oct4, Sox2, and c$M y c$. But, the expression level of $\mathrm{p}^{2} 1^{\mathrm{CIP} 1}$ increases [26]. There is a positive feedback loop between MSI1 and Wnt signaling pathway. Also, there is a functional binding site of $\mathrm{T}$ cell factor/lymphoid enhancer factor (TCF/LEF) transcription factor in MSI1 promoter via in silico analysis. Therefore, the expression of MSI1 could be regulated by activation of Wnt signaling pathway. Following activation of Wnt signaling pathway through enhancement of activated $\beta$-Catenin, expression of target genes like cyclinD1, D2, and myc elevates. On the other hand, by overexpression of MSI1 in intestinal epithelium stem cells, intracellular domain of the Notch-1 receptor (NICD) and Hes1 upregulate and notch signaling pathway is activated [27]. Downregulation of MSI1 in glioblastoma cell lines caused prolongation of cell cycle through accumulation of cyclinB1. In addition, enhancement of Numb and PTEN as a result of MSI reduction

Table 1 Diverse roles of Msi1 in different cell

\begin{tabular}{llll}
\hline Cell type & Function & Mechanisms & References \\
\hline $\begin{array}{l}\text { Mammary stem/ } \\
\text { progenitor cells }\end{array}$ & Cell proliferation & Activation of Notch and Wnt pathways & Imai, 2001; Wang, 2008 \\
$\begin{array}{l}\text { Intestinal epithelium } \\
\text { cells }\end{array}$ & $\begin{array}{l}\text { Controls of cell cycle and cell } \\
\text { proliferation }\end{array}$ & Stabilizing Ccnd1/CCND1, Cdk6/CDK6 and sox4/SOX4 & Cambuli, 2013 \& 2015 \\
$\begin{array}{l}\text { Oligodendrocyte } \\
\text { progenitor cells (OP) }\end{array}$ & $\begin{array}{l}\text { Transition cell fate (Prevention } \\
\text { of differentiation) }\end{array}$ & de-repression of p21WAF1/CIP1 & Dobson, 2008; MacNicol, \\
Germ cell developments & $\begin{array}{l}\text { Spermatogenesis and Oocyte } \\
\text { maturation }\end{array}$ & $\begin{array}{l}\text { Prevention of MSI2 and Erh RNA-binding activity, Activating } \\
\text { translation of ePABP and PABPC1 }\end{array}$ & $\begin{array}{l}\text { Sutherland, 2014 \& 2015; } \\
\text { Cragle, 2019 }\end{array}$ \\
\hline
\end{tabular}


causes deactivation of PI3-Kinase, Akt, and Notch signaling pathways and inhibits cancer cell proliferation in glioblastoma xenograft tumors [28]. Indeed, silencing of MSI1 inhibits Wnt and Notch pathways and decreases spheroid colony formation in lung cancer [29]. Furthermore, in non-small cell lung carcinoma (NSCLC), MSI1 develops lung cancer through activation of the Akt signaling pathway. By ectopic expression of MSI1 in NSCLC cell lines, A549 and H522, phosphorylated Akt is increased and cancer cell proliferation is elevated. After inhibition of the Akt pathway through treating cell lines with MK2206 small molecules, proliferation of cancer cells decreases [30]. MSI1 is also required for tumorigenesis in medulloblastoma and reduction of its expression decreases neurosphere formation, cell proliferation, and tumor growth. Decreasing in Bcl2 and BIII-tubulin expression followed by MSI1 reduction resulted in prevention of differentiation and apoptosis induction [31, 32]. Ribonucleoprotein immunoprecipitation followed by microarray analysis (RIP-chip) indicated that Small GTP Binding Protein, Rac1, Connective Tissue Growth Factor (CTGF), and Syntenin are the major mRNA targets of an MSI1-associated network in U251 glioblastoma cells [32].

Unlike other tumors which progress by metastasis, in glioblastoma the main mechanisms for tumor spread and relapse stage are cell migration and invasion into nearby normal tissues. Invasion depends on intrinsic factors and interaction between cells and extracellular matrix. Gene ontology and pathway analysis have shown that MSI1 has an important role in multiple processes such as cell adhesion, invasion, and migration. Defect in adhesion pathway following MSI1 knockdown can give rise to reduction in translation of related proteins including Platelet-Derived Growth Factor Receptor Alpha (PDGFR $\alpha$ ), Epidermal Growth Factor Receptor (EGFR), and Insulin Growth Factor 1 Receptor (IGF1R). Therefore, in glioblastoma, MSI1 has a major role in migration, adhesion, and invasion pathways by interacting with target genes [33]. Recent study about molecular mechanisms of the cell migration in glioblastoma indicated that MSI1 promotes cell migration by inhibition in translation of Tensin3, a negative regulator of migration [34], and overexpression of Intercellular Adhesion Molecule-1 (ICAM1) [35]. MSI1 could promote tumor progression through interaction with Ago2 in glioblastoma. Under hypoxic stress, MSI1 recruits cytosolic AGO2 and MSI1/AGO2 complex binds to target mRNAs of cell cycle regulators. This complex destabilizes target mRNAs related to apoptosis. Therefore, MSI1/AGO2 pathway promotes stress-induced tumor growth [36].

Furthermore, silencing of MSI1 increases DNA damages in cancer cells treated with ionizing radiation through reduction in frequency of non-homologous end-joining (NHEJ) repair. DNA protein kinase catalytic subunit (DNA-PKcs) has a main role in NHEJ, in which its expression is controlled by MSI1. DNA-PKcs, a main factor in NHEJ, is one of the MSI1 target genes which is stabilized by MSI1 and thereby increases the repair of DNA damage and cell survival [37].

Breast cancer is a heterogeneous disease composed of different subtypes of breast cancer cells with different morphological features and clinical behaviors. One of the most abundant subtypes of breast cancer is estrogen and progesterone receptor positive cells $\left(\mathrm{ER} \alpha / \mathrm{PR}^{+}\right)$[24] $\mathrm{ER} \alpha / \mathrm{PR}^{+}$cells are breast epithelial stem cells that preserve self-renewal through asymmetric cell division. It is revealed that loss of the two main regulators of asymmetric cell division, MSI1 and Notch1, in these cells causes creation of $\mathrm{ER} \alpha / \mathrm{PR}^{+}$breast cancer [38]. Additionally, MSI1 maintains cancer stem cell phenotype as a consequence of stimulating Notch signaling due to downregulation of $26 \mathrm{~s}$ proteasome activity. A conserved nuclear transcription factor $\mathrm{Y}$ (NF-Y) complex consists of three subunits, NF-YA, NF-YB, and NF-YC. NF-YA regulates expression of $26 \mathrm{~s}$ proteasome subunit and in both breast cancer and glioma. MSI1 binds mRNA of this factor and decreases its protein level and activity. Eventually, MSI1 downregulates the expression of $26 \mathrm{~s}$ proteasome subunit [39]. Another mechanism for MSI1 to promote cell growth in breast cancer is stabilizing the target mRNA. In this way, MSI1 stabilizes Tachykinin1 (TAC1) mRNA by competing with miR-130a and miR206. MSI1 binds to noncoding 3'UTR of TAC1 mRNA within exon 7 , therefore causing enhancement of substance $\mathrm{P}$ as its major peptide [40].

Contrary to the role of MSI1 in other tumors which increases cancer cell proliferation, in luminal tumors and luminal breast cancer cell lines, MSI1 is responsible for epithelial-luminal transition. On the other hand, MSI1 inhibits epithelial-mesenchymal transition (EMT) by downregulation of JAGGED1 as a trigger of Notch. Downregulation of MSI1 by RNAi changes the morphology of luminal breast cancer cells (MCF-7) to basallike appearance. In addition, epithelial marker expression is decreased while mesenchymal marker expression increases [25]. Therefore, considering the MSI1 role in breast cancer progression, this gene is essential for the preservation of epithelial-luminal transition.

Although the roles of MSI1 in different cancers extensively correlated with proliferation of cancer cells, its role in cancer cell migration and invasion proffers a much broader role. A recent study has shown a positive correlation between MSI1 and epithelial-mesenchymal markers, vimentin, and snail in tissues of cervical cancer [41]. On the other hand, MSI1 knockdown decreases proliferation of cancer stem cells and induces apoptosis 
in solid tumors. In lung cancer, MSI1 is a diagnostic marker and is highly expressed in spheroid cultures of tumor cells. Knockdown of MSI1 in A549 bronchioalveolar carcinoma and NCI-H520 squamous cell carcinoma reduces the spheroid colony proliferation. This phenomenon is accompanied by a decrease of nuclear localization of $\beta$-catenin. The enhancement of Numb followed by MSI1 knockdown inhibits intracellular processing of Notch. On the other way, MSII silencing in oral squamous cell carcinoma cell lines represses cell proliferation and progression through inactivation of the STAT3 signaling pathway [42]. Reduction of MSI1 expression in breast cancer cell lines decreased the NOTCH1, c-MYC, ERBB2, and ERK1/2 expressions, which eventually inhibited the survival of tumor cells [26]. Downregulation of MSI1 increases apoptosis and G2/M arrest in human colon carcinoma HCT 116 cell line and leads to regression of tumor xenografts [43]. In glioblastoma and medulloblastoma cells, the downregulation of MSI1 induced elongation of mitosis by accumulation of cyclin B1 in addition to the reduction of notch and PI3 kinase-Akt signaling pathways which results in reduction of the cell growth and survival [28].

Overexpression of MSI1 has been observed in treated cancer cells with chemotherapy drugs and radiotherapy. In drug resistance pathway, MSI1 is effective in protection of cancer cells form apoptosis. Following chemotherapy treatment in glioblastoma, enhancement of MSI1 expression causes IL-6 secretion through activation of AKT. Secretion of cytokine IL- 6 proceeds to a positive regulatory loop between IL-6/AKT and enhanced phosphorylation of AKT on serine 473. Following the MSI1/AKT/IL-6 axis, the apoptosis rate of treated cancer cells diminishes through reduction of caspase 3 and cleaved Poly (ADP-ribose) polymerase (PARR) activation [44]. Also, it is revealed that MSI1 induces anti-apoptotic stress granule (SG) formation following 5fluorouracil treatment in colorectal cancers [45]. Recently, it has been shown that MSI1 stimulates glucose uptake in non-small cell lung carcinoma (NSCLC) and influences the mitochondrial respiration and aerobic glycolysis affecting the cell oxygen consumption rate (OCR) and extracellular acidification rate (ECAR) in A549 and H522 cells [30]. In hepatocellular carcinoma, expression of MSI1 elevates. It is already evidenced that overexpression of MSI1 triggers activation of the $\mathrm{Wnt} / \beta$-catenin signaling pathway, thereby increasing migration and invasion of hepatocellular carcinoma cells. On the other hand, stemness properties of cancer stem cells in hepatocellular carcinoma are dependent to MSI1 and CD44 expression [46] (Figs. 1 and 2). A brief description on diverse functions of MSI1 for tumor progression and malignancy is shown in Table 2.

\section{Regulation of MSI1 in different cancers}

The factors that control MSII expression and promote upregulation of the gene in tumors are mostly unknown. Gene expression can be regulated by epigenetic alteration. One of the genes that are highly expressed in breast cancer stem cells is MSI1. CpG-rich sites were
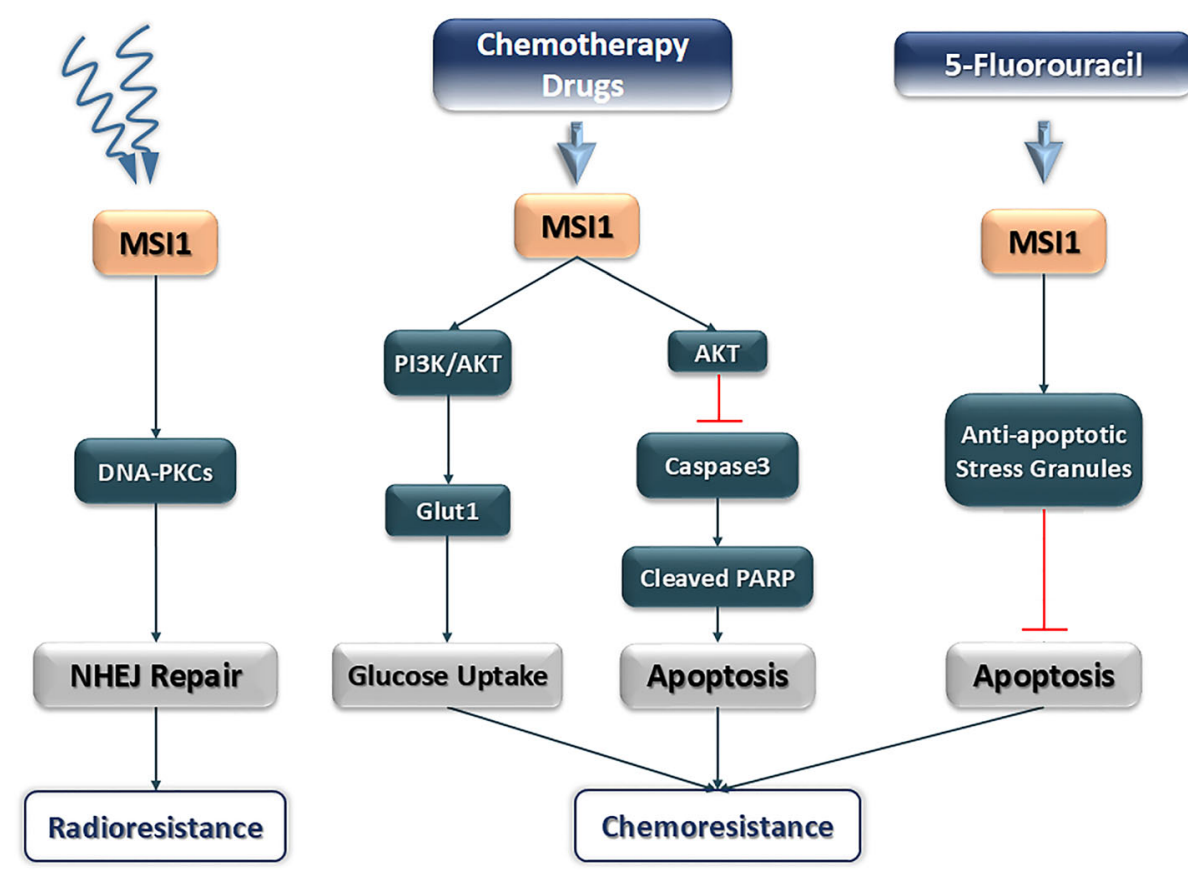

Fig. 2 Different signaling pathways which are mediated by MSI1 upon chemotherapy and radiotherapy approaches in a variety of cancers 
Table 2 The role of MSI1 in promotion of different solid tumors

\begin{tabular}{|c|c|c|c|c|c|}
\hline $\begin{array}{l}\text { Type of } \\
\text { cancer }\end{array}$ & Pathways & Function & Target genes & $\begin{array}{l}\text { Regulation of } \mathbf{M S I} \mathbf{1} \\
\text { expression }\end{array}$ & Reference \\
\hline $\begin{array}{l}\text { Glioblastoma } \\
\text { multiform }\end{array}$ & Notch signaling & $\begin{array}{l}\text { Proliferation of glioblastoma; } \\
\text { tumorspheroids and neuronal } \\
\text { differentiation }\end{array}$ & - & $\begin{array}{l}\text { miR-34a, miR-101, } \\
\text { miR-128, miR-137, } \\
\text { miR-138 }\end{array}$ & $\begin{array}{l}\text { Penalva, } \\
2011\end{array}$ \\
\hline $\begin{array}{l}\text { Glioblastoma } \\
\text { multiform }\end{array}$ & $\begin{array}{l}\text { Notch and PI3 Kinase/Akt } \\
\text { Signaling }\end{array}$ & Cancer cell growth & Caspase3 & - & $\begin{array}{l}\text { Okano, } \\
2012\end{array}$ \\
\hline $\begin{array}{l}\text { Glioblastoma } \\
\text { multiform }\end{array}$ & $\begin{array}{l}\text { Double-strand break repair and } \\
\text { nonhomologous end-joining } \\
\text { (NHEJ) }\end{array}$ & Radio-resistance & $\begin{array}{l}\text { DNA-protein kinase } \\
\text { catalytic subunit (DNA- } \\
\text { PKcs) }\end{array}$ & - & $\begin{array}{l}\text { Penalva, } \\
2016\end{array}$ \\
\hline $\begin{array}{l}\text { Glioblastoma } \\
\text { multiform }\end{array}$ & AKT signaling and apoptosis & Malignancy and chemoresistance & Caspase3, cleaved PARP & - & $\begin{array}{l}\text { Chen, } \\
2016\end{array}$ \\
\hline $\begin{array}{l}\text { Colorectal } \\
\text { cancer }\end{array}$ & Wnt and Notch signaling & Cancer progression & Hes1, c-Myc, APC & miR-137 & $\begin{array}{l}\text { Liang, } \\
2015\end{array}$ \\
\hline $\begin{array}{l}\text { Ovarian } \\
\text { cancer }\end{array}$ & - & Tumor progression & $\mathrm{p} 21, \mathrm{p} 27$, and $\mathrm{p} 53$ & miR-761 & $\begin{array}{l}\text { Zhang, } \\
2016\end{array}$ \\
\hline Lung cancer & AKT signaling & Malignancy and Chemo resistance & PAKT & miR-181 & Xu, 2017 \\
\hline $\begin{array}{l}\text { Cervical } \\
\text { cancer }\end{array}$ & Cell cycle checkpoint & Tumor growth and cell proliferation & $\mathrm{p} 21, \mathrm{p} 27$ and p53 & - & $\begin{array}{l}\text { Zheng, } \\
2014\end{array}$ \\
\hline Breast cancer & Epithelial-mesenchymal transition & Epithelial-luminal cell state & Jagged1 & - & $\begin{array}{l}\text { Burge, } \\
2014\end{array}$ \\
\hline
\end{tabular}

recognized in the promoter region of MSI1 gene. Methylation of $\mathrm{CpG}$ islands in the MSI1 promoter has been shown in several primary breast cancer tumors where hypomethylation leads to gene activation [47]. Different factors which stabilize MSI1 could enhance the cancer progress and drug resistance of cancer cells. Tenascin $C$ (TNC), an extracellular matrix protein expressed in stem cell niches of breast cancer, supports pulmonary metastasis. TNC enhances expression of MSI1 and protects MSI1-dependent Notch signaling by activation of STAT5 [48]. In colorectal cancer cells, the expression of MSI1 is regulated by Notch 3 signaling. Delta-like 4 (DLL)-4 ligand stimulates Notch1 and increases expression level of MSI1. MSI1 as an activator of Notch signaling pathway supports direct activation of Notch1 by DLL4. Activation of Notch1 amplifies transcription of Notch 3 and therefore sustains the circuit [49].

HuR, another important RNA-binding protein is highly expressed in glioblastoma tumors and interacts with 3'UTR of MSI1 which is rich in U and AU sequences. HuR as an important regulator of MSI1 stabilizes MSI1 mRNA and increases its translation. By silencing HuR in U251 and U343 glioblastoma cell lines, the proliferation of cells is reduced and apoptosis is increased, but with ectopic expression of MSI1, apoptosis will be rescued [50].

Some small molecules with inhibition of MSI1 function could facilitate to stop cancer cell proliferation and tumor progression. Gossypol as one of these small molecules could interrupt the binding of MSI1 to target mRNA. Gossypol, a natural phenol, binds to RNAbinding domain1 (RBD1) of MSI1 protein to compete with the target mRNA. Consequently, expression levels of $N U M B$ and $p 21$ increases and Wnt and Notch signaling pathways are downregulated. Treatment of colon cancer cell lines with gossypol inhibits cell proliferation and induces apoptosis. Furthermore, gossypol prevents cancer cell line xenograft tumor growth in mouse models [51]. Luteolin is a type of flavonoid which showed a sturdy direct interaction with MSI1. Proliferation of U251 and U343 GBM (glioblastoma) cell lines treated with luteolin was reduced and the treatment repressed migration and invasion of glioblastoma cells. With knockdown of MSI1 in U251 cells, their sensitivity to luteolin was diminished. So, luteolin is an inhibitor of MSI1 and has an important role in GBM therapy [52].

MicroRNAs are small, noncoding RNAs that control gene expression. MicroRNAs destabilize mRNA by binding to the 3'UTR of target mRNA and hence prevent mRNA translation and protein synthesis [53]. Regulation of some mRNAs at post-translational levels is dependent on polymorphisms at miRNA binding sites in their 3' UTRs. Lately, it was indicated that SNPs' existence at 3' UTR of target gene in place of binding site for microRNAs has an important role in the development of breast cancer. Interaction of microRNAs with 3'UTR of ErbB4 mRNA is more frequent when $\mathrm{T}$ allele at the rs1836724 position is replaced by $\mathrm{C}$ allele [54]. MicroRNAs show oncogenic or tumor suppressor activity in cancerous cells. The expression of some microRNAs are different in several stages of cancer tissues. For instance, miR-9 has an argument expression pattern in different progression levels of breast cancer [55]. Recent data from experimental and computational approaches have 
shown that cancer pathways are not regulated by single microRNAs but are controlled with some networks of multiple microRNAs [56]. 3'UTR length of target genes for microRNA are approximately 1600 nucleotides whereas 3'UTR length of non-miRNA target genes are smaller and their length is about 1000 nucleotides [57].

How microRNAs can perform their functional roles in control of diverse cancers progression through regulation of MSI1 expression will be discussed. MSI1 RNA comprises long 3'UTR (approximately 1800 nucleotides) which could be controlled by several microRNAs. Tumor suppressor miRNAs (miR-34a, miR-101, miR128, miR-137, and miR-138) regulate expression of MSI1 in U251 glioblastoma and Daoy medulloblastoma cells. Transfection of these miRNA mimics into cancer cell lines decrease the cell proliferation. Neuroblastic differentiation assay has shown that after treatment with all-trans retinoic acid and induction of differentiation, expression of MSI1 was reduced. Conversely, a higher expression of aforementioned tumor suppressor miRNAs was detected after differentiation [58]. It was shown that MSI1 is an activator of Wnt and Notch pathways controlled by miR-133a, miR-138, miR-342, miR-491, and miR-541 in colon carcinoma cell lines [59]. Additionally, in the HCT-116 cell line, miR-137 reduced Wnt and notch signaling pathway as oncogenic pathways and was promoted by MSI1. MSI1 as a tumor suppressor declines the growth of human colon cancer xenograft and inhibits colony formation by cancer cells. The expression of miR-137 in rectal cancer tissue was lower and showed that the loss of miR-137 induced a significant expression of MSI1 [60]. In ovarian carcinoma, miR-761 inhibits cancer cell proliferation and invasion. Overexpression of miR-761 increases expression of p21, p27, and p53 that are the target of MSI1 gene [61]. A recent study has shown that MSI1 was overexpressed in gastric cancer cell lines and gastric cancer tissues. MiR-330 regulates MSI1 expression in gastric cancer. Consequently, overexpression of miR-330 in HCG-27 cells led to inhibition of cell proliferation and suppression of colony formation. Cell treatment with histone deacetylase inhibitor, trichostatin A (TSA), and DNA methylation inhibitor 5-aza-CdR (AZA) upregulates expression of miR-330. Hence, regulation of miR-330 expression partly was interceded by hypermethylation of the MSI1 promoter region. As a result, MSI1 was regulated directly by miR-330 in gastric cancer cells [62]. During studies in non-small cell lung carcinoma, it was demonstrated that MSI1 is regulated by miR-181a-5p and there is a negative correlation between MSI1 and miR-181a-5p expressions in NSCLC patients [30]. Nevertheless, an investigation about miRNAs' role in regulation of MSI1 regarding inhibition of some cancers like breast cancer is lacking. Some factors which regulate MSI1 expression in different cancers are represented in Fig. 3.

\section{Regulation of MSI1 in stem cell development}

The role of MSI1 in stem cell development is of interest. Cuadradu and colleagues have confirmed that the thyroid hormone regulates MSII expression in rat brain development. Hypothyroidism in rat causes downregulation of MSI1 expression in glial and neuron cells. After thyroid hormone injection, in vivo upregulation of MSI1 was shown in cerebellum. Also, treatment of neuroblastoma N2a cells with T3 hormone shows that MSI1 amount was increased in the cytoplasm and nucleus. The effect of MSI1 overexpression on brain development following enhancement of T3 hormone is an augmentation for Tau transcript [63]. Furthermore, regulation of MSI1 through thyroid hormone is shown in adult progenitor cell differentiation during amphibian gastrointestinal remodeling [64].

As mentioned, endogenous expression of Msi1 is high in mouse neural stem/progenitor cells. Neural stem cellspecific enhancer is positioned on the sixth intron and is involved in neural development. In mouse neural development, expression of Msi1 is regulated by binding of regulatory transcription factors $\mathrm{x}(\mathrm{RFx})$ to regulatory region of Msi1 which is located in the sixth intron of Msi1 gene $[65,66]$.

\section{Discussion}

MSI1 is a conserved gene during evolution which has a critical role in diverse biological processes such as stem cell proliferation, development, and oncogenesis. MSI1 is essential for proliferation and maintenance of undifferentiated state in neural stem cells. Genome sequencing data in most of cancer tissues compared with normal tissues have shown that MSI1 was not mutated significantly. But, expression of MSI1 is increased in at least $40 \%$ of breast, prostate, and lung tumors and its profile is similar to oncogenes like FOS and HER2. Recently, some evidence represented that MSI1 promotes proliferation and preserves survival of cancer cells in different tumors like glioblastoma, hepatocellular carcinoma, and lung cancer. Enhancement of MSI1 expression causes tumor spread and relapse. Thus, scientists believe that high MSI1 is an indicator of poor survival and poor prognosis [26, 33]. MSI1 is a main therapeutic target because it impacts a broad group of cancers [25]. Chemotherapy is vital for improvement of clinical outcome in cancer patients; however, different studies have shown that MSI1 contributes to chemoresistance in cancer therapy [45]. It was demonstrated that the expression level of MSI1 was amplified followed by cis-diammine dichloroplatinum (CDDP) therapy in A549 and H522 cell lines. On the other hand, MSI1 controls carcinoma 


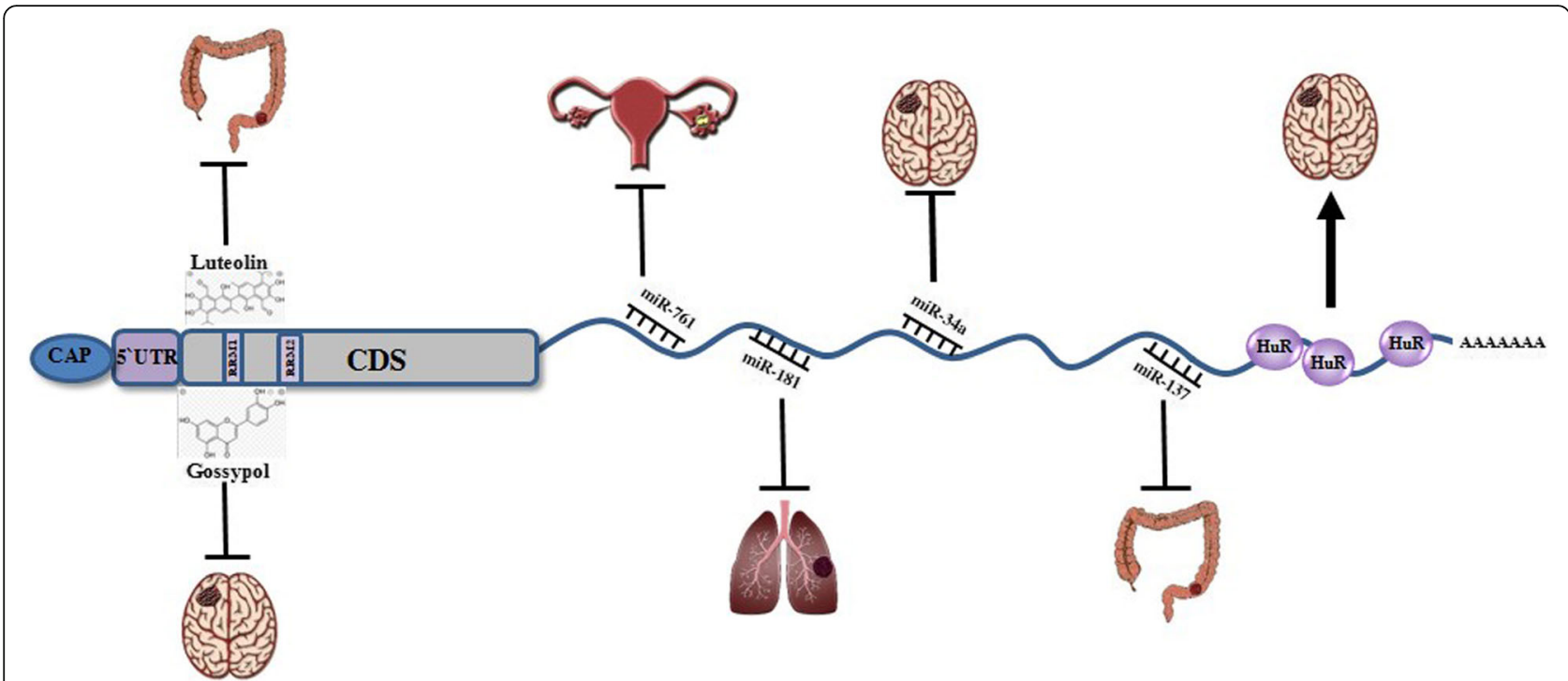

Fig. 3 Schematic representation for the functions of MSI1 in various cancer types. Downregulation of Msi1 by binding of tumor suppressor miRNAs could inhibit the growth of tumor cells in different solid tumors. Furthermore, some small molecules like luteolin and gossypol could interact with RNA binding domain1 (RBD1) in CDS of MS/1 and prevent proliferation of cancer cells and migration. But RNA-binding protein HuR stabilizes MSI1 and promotes gliblastoma by binding to 3'UTR of MSI1

malignancy and chemoresistance through modifying the activity of the Akt signaling pathway in non-small cell lung carcinoma. Inhibition of MSI1 increased the efficiency of radiotherapy and the overexpression of MSI1 induced chemoresistance in glioblastoma [37, 44]. Considering the important role of MSI1 in DNA repair, resistance to chemotherapy, and spread of cancer through normal tissues, MSI1 targeting using different approaches may have the potential application as a reliable strategy for improvement of cancer treatment.

\section{Abbreviations \\ AHR: Aryl hydrocarbon receptor; AZA: DNA methylation inhibitor 5-aza-CdR; CDDP: Cis-diammine dichloroplatinum; CTGF: Connective tissue growth factor; DKK3: Cyclin-dependent kinase inhibitor p21 $1^{\text {Cip1, }}$, Dickkopf-3; DLL- 4: Delta-like 4; DNA-PKcs: DNA protein kinase catalytic subunit; \\ ECAR: Extracellular acidification rate; EGFR: Epidermal growth factor receptor; EMT: Epithelial-mesenchymal transition; ePABP: Embryonic the poly (A) binding protein family; Erh: Enhancer of rudimentary homolog; \\ HCC: Hepatocellular carcinoma; HSPC: Hematopoietic stem and progenitor cell; ICAM1: Intercellular adhesion molecule-1; IGF1R: Insulin growth factor1 receptor; Msi1: Musashi1; NICD: Intracellular domain of the Notch-1 receptor; NF-YA: Nuclear transcription factor Y subunit alpha; NHEJ: Nonhomologous end-joining; NSCLC: Non-small cell lung carcinoma; NSPCs: Neural stem/ progenitor cells; OCR: Oxygen consumption rate; PABP: Poly (A) binding protein; PABPC1: Canonical somatic cell poly(A) binding protein; PARP: Poly (ADP-ribose) polymerase; PDGFRa: Platelet-derived growth factor receptor alpha; RBD: RNA-binding domain; RFX transcription factors: Regulatory factor $X$ transcription factors; RIP-chip: Ribonucleoprotein immunoprecipitation followed by microarray analysis; rISCs: Reserve intestinal stem cells; SGs: Stress granules; SOP: Sensory organ precursor; TAC1: Tachykinin1; TCF/ LEF: T cell factor/lymphoid enhancer factor; TNC: Tenascin C; TSA: Trichostatin A}

\section{Acknowledgements}

We sincerely acknowledge our colleagues at the Royan Institute and University of Isfahan for helpful discussion and suggestions.

\section{Authors' contributions}

The design of study was done by M.F., K.D., M.H.N.E., and K.G.. Data mining was performed by M.F, L.L.. Interpretation of the obtained information was done by all authors. The manuscript was written by M.F., A.O.G., and K.G. and approved by all authors.

\section{Funding}

No funding to report.

\section{Availability of data and materials \\ Not applicable.}

Ethics approval and consent to participate

Not applicable.

\section{Consent for publication}

Not applicable.

\section{Competing interests}

The authors declare that they have no competing interests.

\section{Author details}

'Department of Cell and Molecular Biology and Microbiology, Faculty of Biological Science and Technology, University of Isfahan, Hezar Jerib Ave. Azadi Square, Isfahan P.O. Code 81746, Iran. ${ }^{2}$ Department of Molecular Biotechnology, Cell Science Research Center, Royan Institute for Biotechnology, ACECR, Isfahan P.O. Code 816513-1378, Iran. ${ }^{3}$ Department of Cellular Biotechnology, Cell Science Research Center, Royan Institute for Biotechnology, ACECR, Isfahan, Iran. ${ }^{4}$ Department of Molecular Biology and Genetics, Faculty of Science, Bilkent University, Ankara, Turkey.

Received: 25 February 2020 Revised: 31 March 2020

Accepted: 5 May 2020 Published online: 24 May 2020

\section{References}

1. Nakamura M, Okano H, Blendy JA, Montell C. Musashi, a neural RNA-binding protein required for Drosophila adult external sensory organ development. Neuron. 1994;13(1):67-81.

2. Okano H, Imai T, Okabe M. Musashi: a translational regulator of cell fate. J Cell Sci. 2002;115(7):1355-9. 
3. S-i S, Imai T, Hamaguchi K, et al. Mouse-Musashi-1, a neural RNA-binding protein highly enriched in the mammalian CNS stem cell. Dev Biol. 1996; 176(2):230-42.

4. S-i S, Nakamura Y, Satoh H, Okano H. Rna-binding protein Musashi2: developmentally regulated expression in neural precursor cells and subpopulations of neurons in mammalian CNS. J Neurosci. 2001;21(20): 8091-107.

5. Taggart J, Ho T-C, Amin E, et al. MSI2 is required for maintaining activated myelodysplastic syndrome stem cells. Nat Commun. 2016;7:10739.

6. Good P, Yoda A. Sakakibara S-i, et al. the HumanMusashi homolog 1 (MSI1) gene encoding the homologue of Musashi/Nrp-1, a neural RNA-binding protein putatively expressed in CNS stem cells and neural progenitor cells. Genomics. 1998;52(3):382-4.

7. Kaneko Y, Sakakibara S, Imai T, et al. Musashi1: an evolutionally conserved marker for CNS progenitor cells including neural stem cells. Dev Neurosci. 2000;22(1-2):139-53.

8. Okabe M, Imai T, Kurusu M, Hiromi Y, Okano H. Translational repression determines a neuronal potential in Drosophila asymmetric cell division. Nature. 2001;411(6833):94-8.

9. Kawahara H, Imai T, Imataka H, Tsujimoto M, Matsumoto K, Okano H. Neural RNA-binding protein Musashi1 inhibits translation initiation by competing with elF4G for PABP. J Cell Biol. 2008;181(4):639-53.

10. Imai T, Tokunaga A, Yoshida T, et al. The neural RNA-binding protein Musashi1 translationally regulates mammalian numb gene expression by interacting with its mRNA. Mol Cell Biol. 2001;21(12):3888-900.

11. Glazer RI, Vo DT, Penalva L. Musashi1: an RBP with versatile functions in normal and cancer stem cells. Front Biosci. 2012;17(1):54-64.

12. Liu X, Yang W-T, Zheng P-S. Msi1 promotes tumor growth and cell proliferation by targeting cell cycle checkpoint proteins p21, p27 and p53 in cervical carcinomas. Oncotarget. 2014;5(21):10870.

13. Wang $X-Y$, Yin $Y$, Yuan H, Sakamaki T, Okano H, Glazer RI. Musashi1 modulates mammary progenitor cell expansion through proliferinmediated activation of the Wnt and notch pathways. Mol Cell Biol. 2008;28(11):3589-99.

14. Dobson NR, Zhou YX, Flint NC, Armstrong RC. Musashi1 RNA-binding protein regulates oligodendrocyte lineage cell differentiation and survival. Glia. 2008;56(3):318-30.

15. MacNicol AM, Hardy LL, Spencer HJ, MacNicol MC. Neural stem and progenitor cell fate transition requires regulation of Musashi1 function. BMC Dev Biol. 2015;15(1):1.

16. Cambuli F, Correa B, Rezza A, et al. A mouse model of targeted Musashi1 expression in whole intestinal epithelium suggests regulatory roles in cell cycle and stemness. Stem Cells. 2015;33(12):3621-34.

17. Maria Cambuli F, Rezza A, Nadjar J, Plateroti M. Brief report: Musashi1-eGFP mice, a new tool for differential isolation of the intestinal stem cell populations. Stem Cells. 2013;31(10):2273-8.

18. Orzechowska EJ, Parajuli S, Bialkowska AB, Yang W. Mo1065-P21 Waf1/Cip1 and RNA binding protein Musashi-1 regulate small intestinal epithelium regeneration after $\Gamma$ radiation-induced injury by activation of the subpopulation of reserve intestinal stem cells in vivo. Gastroenterology. 2018;154(6):S-688.

19. Sutherland JM, Sobinoff AP, Fraser BA, et al. RNA binding protein Musashi-1 directly targets Msi2 and Erh during early testis germ cell development and interacts with IPO5 upon translocation to the nucleus. FASEB J. 2015;29(7): 2759-68.

20. Sutherland JM, Fraser BA, Sobinoff AP, et al. Developmental expression of Musashi-1 and Musashi-2 RNA-binding proteins during spermatogenesis: analysis of the deleterious effects of dysregulated expression. Biol Reprod. 2014;90(5):92.

21. Cragle CE, MacNicol MC, Byrum SD, et al. Musashi interaction with poly (A) binding protein is required for activation of target mRNA translation. J Bio Chem. 2019;jbc(RA119):007220.

22. Rentas S, Holzapfel NT, Belew MS, et al. Musashi-2 attenuates AHR signalling to expand human haematopoietic stem cells. Nature. 2016;532(7600):508-11.

23. Fang $T, L v H, W u$ F, et al. Musashi 2 contributes to the stemness and chemoresistance of liver cancer stem cells via LIN28A activation. Cancer Lett. 2017;384:50-9.

24. Network CGA. Comprehensive molecular portraits of human breast tumours. Nature. 2012;490(7418):61-70.

25. Katz Y, Li F, Lambert NJ, et al. Musashi proteins are post-transcriptional regulators of the epithelial-luminal cell state. Elife. 2014;3:e03915.
26. Wang $X-Y$, Penalva LO, Yuan $H$, et al. Musashi1 regulates breast tumor cell proliferation and is a prognostic indicator of poor survival. Mol Cancer. 2010;9(1):1.

27. Rezza A, Skah S, Roche C, Nadjar J, Samarut J, Plateroti M. The overexpression of the putative gut stem cell marker Musashi-1 induces tumorigenesis through Wnt and notch activation. J Cell Sci. 2010;123(19): 3256-65.

28. Muto J, Imai T, Ogawa D, et al. RNA-binding protein musashi1 modulates glioma cell growth through the post-transcriptional regulation of notch and PI 3 kinase/Akt signaling pathways. PLoS One. 2012;7(3):e33431.

29. Wang $X-Y, Y u H$, Linnpoila Rl, et al. Musashi1 as a potential therapeutic target and diagnostic marker for lung cancer. Oncotarget. 2013;4(5):739-50.

30. Lang $Y$, Kong $X, \mathrm{He} C$, et al. Musashi1 promotes non-small cell lung carcinoma malignancy and chemoresistance via activating the Akt signaling pathway. Cell Physiol Biochem. 2017:44(2):455-66.

31. Sanchez-Diaz PC, Burton TL, Burns SC, Hung JY, Penalva LO. Musashi1 modulates cell proliferation genes in the medulloblastoma cell line Daoy. BMC Cancer. 2008;8(1):1.

32. Vo DT, Subramaniam D, Remke M, et al. The RNA-binding protein Musashi1 affects medulloblastoma growth via a network of cancer-related genes and is an indicator of poor prognosis. Am J Pathol. 2012;181(5):1762-72.

33. Uren PJ, Vo DT, de Araujo PR, et al. RNA-binding protein Musashi1 is a central regulator of adhesion pathways in glioblastoma. Mol Cell Biol. 2015; 35(17):2965-78

34. Chen $\mathrm{H}-\mathrm{Y}$, Lin $\mathrm{L}-\mathrm{T}$, Wang $\mathrm{M}-\mathrm{L}$, et al. Musashi-1 enhances glioblastoma cell migration and cytoskeletal dynamics through translational inhibition of Tensin3. Sci Rep. 2017;7

35. Lin J-C, Tsai J-T, Chao T-Y, Ma H-I, Liu W-H. Musashi-1 enhances glioblastoma migration by promoting ICAM1 translation. Neoplasia. 2019; 21(5):459-68.

36. Chen $\mathrm{H}-\mathrm{Y}$, Wang $\mathrm{M}-\mathrm{L}$, Laurent $\mathrm{B}$, et al. Musashi-1 promotes stress-induced tumor progression through recruitment of AGO2. THERANOSTICS. 2020; 10(1):201-17.

37. de Araujo PR, Gorthi A, da Silva AE, et al. Musashi1 impacts radio-resistance in glioblastoma by controlling DNA-protein kinase catalytic subunit. Am J Pathol. 2016;186(9):2271-8.

38. Clarke RB, Anderson E, Howell A, Potten CS. Regulation of human breast epithelial stem cells. Cell Prolif. 2003;36(s1):45-58.

39. Lagadec C, Vlashi E, Frohnen P, Alhiyari Y, Chan M, Pajonk F. The RNAbinding protein Musashi-1 regulates proteasome subunit expression in breast cancer-and glioma-initiating cells. Stem Cells. 2014;32(1):135-44.

40. Nahas GR, Murthy RG, Patel SA, Ganta T, Greco SJ, Rameshwar P. The RNAbinding protein Musashi 1 stabilizes the oncotachykinin 1 mRNA in breast cancer cells to promote cell growth. FASEB J. 2016;30(1):149-59.

41. Gong P, Wang Y, Gao Y, et al. Msi1 promotes tumor progression by epithelialto-mesenchymal transition in cervical cancer. Hum Pathol. 2017;65:53-61.

42. Wang CF, Zhang HC, Feng XM, Song XM, Wu YN. Knockdown of MSI1 inhibits the proliferation of human oral squamous cell carcinoma by inactivating STAT3 signaling. Int J Mol Med. 2019:44(1):115-24.

43. Sureban SM, May R, George RJ, et al. Knockdown of RNA binding protein musashi-1 leads to tumor regression in vivo. Gastroenterology. 2008;134(5): 1448-58. e2.

44. Chen $\mathrm{H}-\mathrm{Y}$, Lin L-T, Wang M-L, et al. Musashi-1 regulates AKT-derived IL-6 autocrinal/paracrinal malignancy and chemoresistance in glioblastoma. Oncotarget. 2016;7(27):42485

45. Chiou G-Y, Yang T-W, Huang C-C, et al. Musashi-1 promotes a cancer stem cell lineage and chemoresistance in colorectal cancer cells. Sci Rep. 2017; 7(1):2172.

46. Liu Q, Zhou C, Zhang B. Upregulation of musashi1 increases malignancy of hepatocellular carcinoma via the Wnt/ $\beta$-catenin signaling pathway and predicts a poor prognosis. BMC Gastroenterol. 2019;19(1):1-10.

47. Kagara N, Huynh KT, Kuo C, et al. Epigenetic regulation of cancer stem cell genes in triple-negative breast cancer. Am J Pathol. 2012;181(1):257-67.

48. Oskarsson T, Acharyya S, Zhang XH, et al. Breast cancer cells produce tenascin $C$ as a metastatic niche component to colonize the lungs. Nat Med. 2011;17(7):867-74.

49. Pastò $A$, Serafin $V$, Pilotto $G$, et al. NOTCH3 signaling regulates MUSASHI-1 expression in metastatic colorectal cancer cells. Cancer Res. 2014;74(7):2106-18.

50. Vo DT, Abdelmohsen K, Martindale $J$, et al. The oncogenic RNA-binding protein Musashi1 is regulated by HuR via mRNA translation and stability in glioblastoma cells. Mol Cancer Res. 2012;10(1):143-55. 
51. Lan L, Appelman C, Smith AR, et al. Natural product (-)-gossypol inhibits colon cancer cell growth by targeting RNA-binding protein Musashi-1. Mol Oncol. 2015;9(7):1406-20.

52. Yi C, Li G, Ivanov DN, et al. Luteolin inhibits Musashi1 binding to RNA and disrupts cancer phenotypes in glioblastoma cells. RNA Biol. 2018;15(11): 1420-32.

53. Bartel DP. MicroRNAs: target recognition and regulatory functions. Cell. 2009;136(2):215-33.

54. Bagheri F, Mesrian Tanha H, Mojtabavi Naeini M, Ghaedi K, Azadeh M. Tumor-promoting function of single nucleotide polymorphism rs 1836724 (C3388T) alters multiple potential legitimate microRNA binding sites at the 3'-untranslated region of ErbB4 in breast cancer. Mol Med Rep. 2016;13(5): 4494-8.

55. Hasanzadeh A, Tanha HM, Ghaedi K, Madani M. Aberrant expression of miR-9 in benign and malignant breast tumors. Mol Cell Probes. 2016:30(5):279-84.

56. Bracken CP, Scott HS, Goodall GJ. A network-biology perspective of microRNA function and dysfunction in cancer. Nat Rev Genet. 2016;17(12): 719-32.

57. Hu Z. Insight into microRNA regulation by analyzing the characteristics of their targets in humans. BMC Genomics. 2009;10(1):594.

58. Vo DT, Qiao M, Smith AD, Burns SC, Brenner AJ, Penalva LO. The oncogenic RNA-binding protein Musashi1 is regulated by tumor suppressor miRNAs. RNA Biol. 2011;8(5):817-28.

59. Hrdličková R, Nehyba J, Bargmann W, Bose HR Jr. Multiple tumor suppressor microRNAs regulate telomerase and TCF7, an important transcriptional regulator of the Wnt pathway. PLoS One. 2014;9(2):e86990.

60. Smith AR, Marquez RT, Tsao W-C, et al. Tumor suppressive microRNA-137 negatively regulates Musashi-1 and colorectal cancer progression Oncotarget. 2015;6(14):12558

61. Shi C, Zhang Z. miR-761 inhibits tumor progression by targeting MSI1 in ovarian carcinoma. Tumor Biol. 2016;37(4):5437-43.

62. Guan A, Wang H, Li X, et al. MiR-330-3p inhibits gastric cancer progression through targeting MSI1. Am J Transl Res. 2016;8(11):4802.

63. Cuadrado A. García-Fernández LF, Imai T, Okano H, Muñoz A. Regulation of tau RNA maturation by thyroid hormone is mediated by the neural RNAbinding protein musashi-1. Mol Cell Neurosci. 2002;20(2):198-210.

64. Ishizuya-Oka A, Shimizu K, Sakakibara S-i, Okano H, Ueda S. Thyroid hormone-upregulated expression of Musashi-1 is specific for progenitor cells of the adult epithelium during amphibian gastrointestinal remodeling. J Cell Sci. 2003;116(15):3157-64.

65. Kawase S, Imai T, Miyauchi-Hara C, et al. Identification of a novel intronic enhancer responsible for the transcriptional regulation of musashi1 in neural stem/progenitor cells. Molecular Brain. 2011;4(1):14.

66. Kawase $\mathrm{S}$, Kuwako $\mathrm{K}$, Imai $\mathrm{T}$, et al. Regulatory factor $\mathrm{X}$ transcription factors control Musashi1 transcription in mouse neural stem/progenitor cells. Stem Cells Dev. 2014;23(18):2250-61.

\section{Publisher's Note}

Springer Nature remains neutral with regard to jurisdictional claims in published maps and institutional affiliations. 